\title{
Nearly $100 \%$ Horizontal Dipole Orientation and Upconversion Efficiency in Blue Thermally Activated Delayed Fluorescent Emitters
}

\author{
Sung Yong Byeon, Jongchan Kim, Dong Ryun Lee, Si Hyun Han, Stephen R. Forrest,* \\ and Jun Yeob Lee*
}

The relationship between anisotropic orientation and molecular structure of thermally activated delayed fluorescent (TADF)-based organic light emitting devices (OLEDs) is studied using TADF emitters with carbazole, biscarbazole, and triscarbazole donor units. The bicarbazole and triscarbazole donors are more effective than the carbazole donor in driving the anisotropic orientation of the TADF molecules. A near-perfect in-plane orientation of the TADF dipole moment is demonstrated using the triscarbazole donor. In addition, the triscarbazole donor based OLED shows high photoluminescence quantum yield and an upconversion efficiency close to $100 \%$. As a consequence, an external quantum efficiency $>30 \%$ is obtained.

strong donor. ${ }^{[12-15]}$ These groups narrow the singlet-triplet energy gap $\left(\Delta E_{\mathrm{ST}}\right)$ and thus lead to enhanced efficiency in green and blue TADF emitters. The donors have also shown a tendency for their molecular transition dipole moments to preferentially align in the in-plane (horizontal) direction relative to the substrate. ${ }^{[16-18]}$ For example, 2,4-bis\{3-(9H-carbazol9-yl)-9H-carbazol-9-yl\}-6-phenyl-1,3,5triazine (CC2TA) which features the carbazolylcarbazole donor shows a preferred horizontal dipole orientation in vacuum-deposited CC2TA:DPEPO (bis [2-(diphenylphosphino)phenyl]

\section{Introduction}

Recently there has been significant progress in improving the external quantum efficiency (EQE) of thermally activated delayed fluorescent (TADF) devices since the demonstration of $\mathrm{EQE} \approx 20 \%$ in organic light-emitting diodes (OLEDs). ${ }^{[1-5]}$ Today, the highest EQE of red, green and blue TADF OLEDs currently stand at $17.5 \%, 31.2 \%$, and $36.7 \%$, respectively. ${ }^{[2,6,7]}$ In the development of high efficiency TADF OLEDs, advances in device performance were mainly achieved by engineering the donor and the acceptor groups of the emitting molecules. ${ }^{[8-10]}$ The combination of strong donors and acceptors has resulted in the complete harvesting of all excitons generated in both singlet and triplet excited states. ${ }^{[11]}$ Representative donor moieties such as dimethylacridine, dicarbazolylcarbazole, di(diphenylaminocarbazole) efficiently harvest the excited states. Carbazole-based donor moieties have a strong donor character by combining multiple donor units, and the dimethylacridine moiety is an intrinsically

S. Y. Byeon, D. R. Lee, S. H. Han, Prof. J. Y. Lee

School of Chemical Engineering

Sungkyunkwan University

2066, Seobu-ro, Jangan-gu, Suwon, Gyeonggi 440-746, South Korea

E-mail: leej17@skku.edu

J. Kim, Prof. S. R. Forrest

Departments of Electrical Engineering and Computer Science

Physics and Materials Science and Engineering

University of Michigan

2238 EECS, Ann Arbor, MI 48109, USA

E-mail: stevefor@umich.edu

DOI: 10.1002/adom.201701340 ether oxide) films. ${ }^{[16,19]}$ Also, dimethylacridine-derived 10-(4-(4,6-diphenyl-1,3,5-triazin-2-yl)phenyl)-9,9-dimethyl9,10-dihydroacridine (DMAC-TRZ) has also shown horizontal alignment. ${ }^{[20]}$ However, correlations between the molecular structure and the dipole orientation was not established. [21-23] Furthermore, the CC2TA emitter showed low external quantum efficiency (EQE) despite its horizontal dipole orientation. ${ }^{[16]}$

Carbazole, biscarbazole, and triscarbazole were adopted as donor units in TADF emitters to study effects of the donor on dipole orientation. We demonstrate that carbazole cannot induce dipole orientation of the TADF emitters, whereas biscarbazole and triscarbazole can. The EQE of OLEDs employing rod-like triscarbazole donor-derived emitters achieve EQE > 30\% due to their near-perfect in-plane orientation. The strong donor character of the triscarbazole results in a uniform distribution of the electron density in the highest occupied molecular orbital, and hence a high photoluminescence (PL) quantum yield. Furthermore, the donor structure leads to a relatively short delayed fluorescence lifetime leading to efficient conversion of triplet to singlet excitons. To our knowledge, this work presents the first demonstration of the simultaneous achievement of nearly $100 \%$ horizontal dipole orientation and $100 \%$ exciton conversion efficiency in TADF-based OLEDs.

\section{Results and Discussion}

Three carbazole-based compounds studied were: 9-(4-(4,6-diphenyl-1, 3, 5-triazin-2-yl)phenyl)- $9 \mathrm{H}$-carbazole (CzTrz), 9-(4-(4,6-diphenyl-1,3,5-triazin-2-yl) phenyl)-9'-phenyl-9H, $9^{\prime} H$-3,3'-bicarbazole (BCzTrz), and 
Table 1. Selected properties of CzTrz, BCzTrz, and TCzTrz.

\begin{tabular}{|c|c|c|c|c|c|c|c|c|c|c|c|}
\hline & $\lambda_{\text {abs }}[\mathrm{nm}]$ & $E_{\mathrm{g}}[\mathrm{eV}]$ & $E_{\mathrm{S}}{ }^{\text {a) }}[\mathrm{eV}]$ & $E_{\mathrm{T}}^{\mathrm{b})}[\mathrm{eV}]$ & $\Delta E_{\mathrm{ST}}[\mathrm{eV}]$ & HOMO/ LUMOc) [eV] & $\Phi^{\mathrm{d})}[\%]$ & $\tau^{\mathrm{e})}[\mu \mathrm{s}]$ & $k_{\mathrm{ISC}} \mathrm{f}^{f}$ & $k_{\mathrm{RISC}}{ }^{\mathrm{f}}$ & $\Theta_{\mid /}{ }^{g)}[\%]$ \\
\hline CzTrz & $265 / 360$ & 3.14 & 3.18 & 2.82 & 0.36 & $-6.04 /-3.39$ & $71(68)$ & $\mathrm{N} / \mathrm{A}$ & $\mathrm{N} / \mathrm{A}$ & N/A & 66 \\
\hline BCzTrz & $265 / 372$ & 2.94 & 2.83 & 2.79 & 0.04 & $-5.80 /-3.24$ & $96(70)$ & 31.2 & $2.46 \times 10^{7}$ & $5.49 \times 10^{4}$ & 89 \\
\hline TCzTrz & $273 / 385$ & 2.84 & 2.73 & 2.72 & 0.01 & $-5.75 /-3.41$ & $99(76)$ & 23.6 & $2.22 \times 10^{7}$ & $5.34 \times 10^{4}$ & 95 \\
\hline
\end{tabular}

a) Singlet energy derived from onset of the fluorescence spectra of $10 \mathrm{wt} \%$ TADF emitter-doped DPEPO films; b) Triplet energy derived from onset of the phosphorescence spectra of 10 wt\% TADF emitter-doped DPEPO films at $77 \mathrm{~K}$; $\left.{ }^{c}\right) \mathrm{HOMO}$ and LUMO energies using cyclic voltammetry, with internal standard of ferrocene; ${ }^{d)} \mathrm{Absolute}$ PL quantum yield measured under nitrogen and oxygen atmosphere; e) Excited state lifetime for $10 \mathrm{wt} \%$ TADF emitter-doped DPEPO films at room temperature; ${ }^{f)} \mathrm{Calculated}$ rate constant derived from $10 \mathrm{wt} \%$ emitter doped DPEPO films; ${ }^{\text {g) }}$ Horizontal orientation ratio for $10 \mathrm{wt} \%$ TADF emitter-doped DPEPO films.

9'-(4-(4,6-diphenyl-1,3,5-triazin-2-yl)phenyl)-9,9"-diphenyl$9 H, 9^{\prime} H, 9^{\prime \prime} H-3,3^{\prime}: 6^{\prime}, 3^{\prime \prime}$-tercarbazole (TCzTrz). Selected material properties are listed in Table 1 , and a detailed analysis is found in Supporting Information (Figures S1 and S2, Supporting Information). TCzTrz was synthesized following procedures described in the Experimental Section, while the other compounds were prepared as previously reported. ${ }^{[30]}$

The geometric structures and transition dipole moments of the emitters are critical to their ability to orient in the thin film. ${ }^{[24,25]}$ A rod-like extended molecular structure or planar molecular backbone induces orientation of the dipole moment in the substrate plane. ${ }^{[2,27]}$ The three emitting molecules contain different donor moieties that are compared in Figure 1. The dihedral angles between the donor and the phenyl link between the donor and acceptor moieties were $51.8^{\circ}, 50.7^{\circ}$, and $50.1^{\circ}$ for CzTrz, BCzTrz, and TCzTrz, respectively, indicating similar distortion of the donor unit in all three molecules. In the case of the BCzTrz and TCzTrz, the dihedral angles between the carbazole units were $39.4^{\circ}$, and $38.6^{\circ}$, indicating the presence of a small distortion between the carbazole units. Therefore, BCzTrz and TCzTrz have an extended donor structure that may induce anisotropic horizontal orientation, as found in other fluorescent molecules. ${ }^{[17]}$

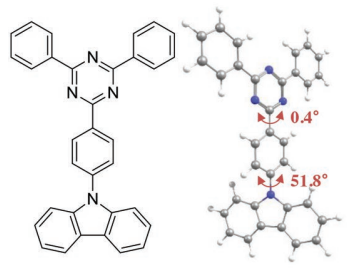

CzTrz
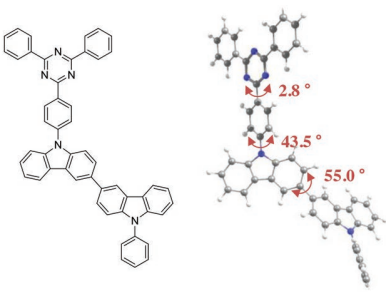

BCzTrz

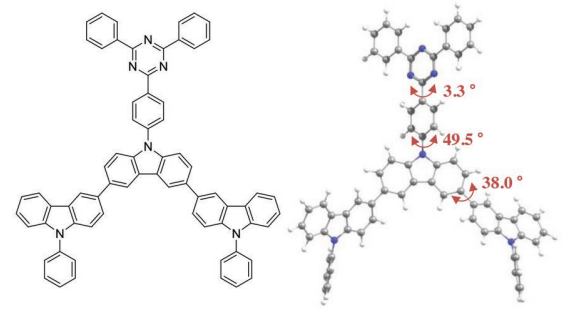

TCzTrz
Figure 1. Molecular and geometrical structures of the CzTrz, BCzTrz, and TCzTrz optimized by density functional theory simulations. Dihedral angles of the chemical bonds are noted in the geometrical structures.
The transition dipole moments of the optimized geometries of the three compounds were calculated using time dependent density functional theory based on the B3LYP 6-31 basis set. CzTrz, BCzTrz, and TCzTrz showed dipole moments preferentially oriented along the molecular axis of the donor-acceptor structure perpendicular to the carbazole unit (see Figure 2). Thus, the transition dipoles lie in the same plane as the donor units.

Figure 3 shows the dipole alignment measurements of the three compounds analyzed using angle-dependent PL. ${ }^{16]}$ The emitters were doped in DPEPO at a concentration of $10 \mathrm{wt} \%$. The horizontal-to-vertical dipole alignment ratios relative to the substrate of TCzTrz, BCzTrz, and CzTrz were $\theta_{\text {hor }}=0.95 \pm$ $0.02,0.89 \pm 0.02$, and $0.66 \pm 0.01$, respectively. ${ }^{[13]}$ The order of the horizontal dipole ratio was, therefore: TCzTrz > BCzTrz > CzTrz, indicating that the triscarbazole and bicarbazole donor moieties lead to preferential in-plane alignment compared to carbazole due to the rod-like extended molecular structures of the former donor units. As the transition dipole moment of the emitter lies in the acceptor plane and the donor is in the same plane, the rod-like donors induce the anisotropic orientation of the emitters. ${ }^{[16]}$

Figure 4 shows the EQE of the CzTrz, BCzTrz, and TCzTrzbased OLEDs with the emitters doped at $10 \mathrm{wt} \%$ in DPEPO. The rather high $10 \mathrm{wt} \%$ doping concentration was used since carriers are balanced by hole hopping between the emitters.
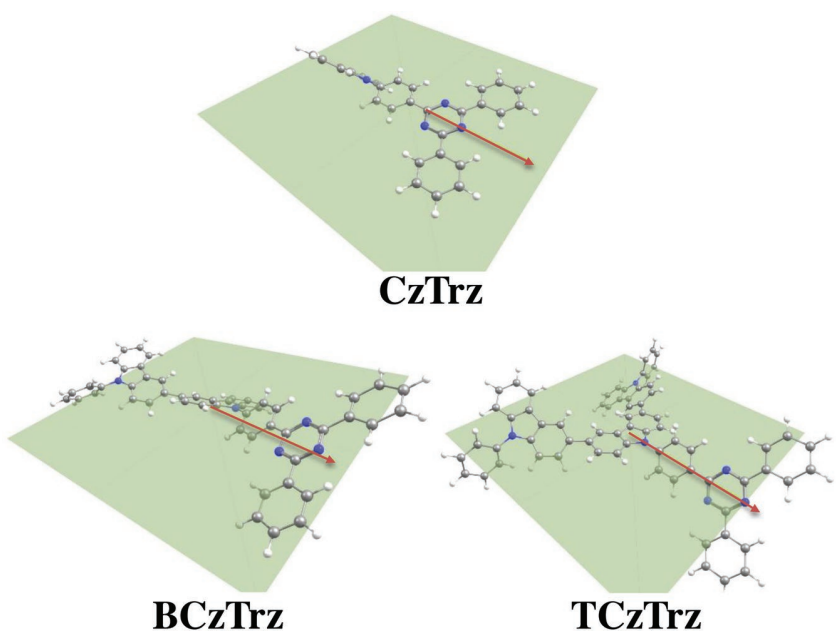

Figure 2. Direction and relative strengths (from arrow lengths, Table 1) of the transition dipole moments of CzTrz, BCzTrz, and TCzTrz calculated by density functional theory. 

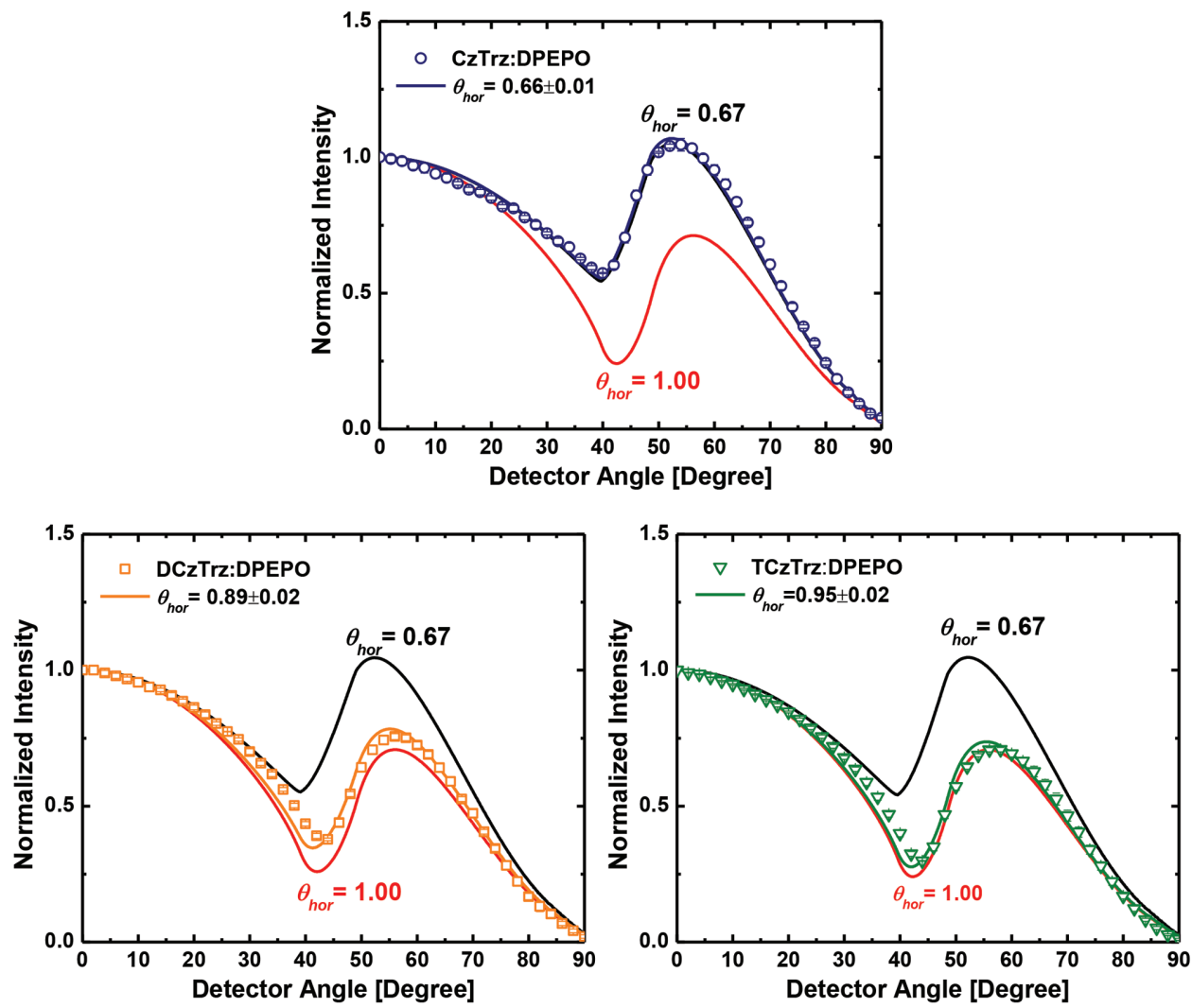

Figure 3. Measured horizontal transition dipole moment ratios of a) $\mathrm{Cz} \operatorname{Trz}$, b) BCzTrz, and c) TCzTrz (data points) obtained using angle-dependent PL data and calculated by optical simulation (lines). Simulated angle-dependent photoluminescence intensity of isotropic and horizontally oriented emitters dipoles are also shown.

Current density, voltage, and luminance data are presented in Figure S3 of the Supporting Information. The maximum EQE of the CzTrz-, BCzTrz-, and TCzTrz-based devices were $4.2 \%$ $\pm 0.04 \%, 23.6 \% \pm 0.24 \%$, and $31.8 \% \pm 0.31 \%$, respectively. As expected, a relatively high EQE was obtained in the TCzTrzbased OLED compared with the CzTrz device. In general, the EQE of the TADF devices is a function of the PL quantum yield, horizontal dipole ratio, and triplet upconversion efficiency.

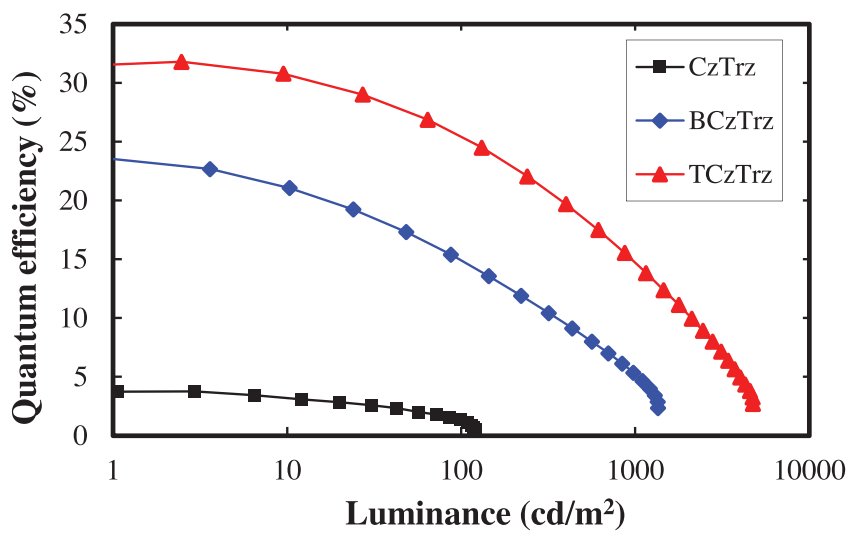

Figure 4. External quantum efficiency vs. luminance of a) $\mathrm{Cz} \operatorname{Trz}$, b) BCzTrz, and c) TCzTrz OLEDs at a TADF doping concentration of $10 \mathrm{wt} \%$ in DPEPO.
The PL quantum yields of CzTrz, BCzTrz, and TCzTrz under a $\mathrm{N}_{2}$ atmosphere were $0.71 \pm 0.01,0.96 \pm 0.02$, and $0.99 \pm 0.02$, respectively, which follows the order of EQE of the OLEDs. From the PL quantum yield under an oxygen/nitrogen ratio of CzTrz (0.68/0.71), BCzTrz (0.70/0.96), and TCzTrz (0.76/0.99) in Table 1, the upconversion efficiencies of triplet excitons in the three emitters were calculated to be $9.5 \% \pm 1.9 \%, 88 \% \pm 5.3 \%$, and $96 \% \pm 4.6 \%$, respectively. ${ }^{[13]}$ The high upconversion efficiency of the TCzTrz is due the short delayed fluorescence lifetime as presented in Table 1. For CzTrz material, very little delayed fluorescence was observed since the carbazole donor does not lead to a significant reduction in $\Delta E_{\mathrm{ST}}$

Combining the PL quantum yield, the upconversion efficiency, and out-coupling factor related to the horizontal dipole orientation (Figure 5), the EQE for the three OLEDs was calculated based on the dyadic Green's function analysis. ${ }^{[28,29]}$ The maximum calculated EQEs of the CzTrz, BCzTrz, and TCzTrz devices were $9.0 \% \pm 0.6 \%, 27.3 \% \pm 1.3 \%$, and $35.4 \% \pm 1.3 \%$, respectively. Although there is some discrepancy between the calculated and measured EQEs possibly due to electrical losses not accounted for in the simulations, the EQEs were nevertheless similar to within experimental and theoretical accuracies.

Electroluminescence spectra of the devices are shown in Figure 6. Blue emission spectra were observed and the order of peak wavelengths was TCzTrz (496 nm) > BCzTrz (485 nm) > CzTrz (449 nm). Color coordinates of the CzTrz, BCzTrz, and 
(a)

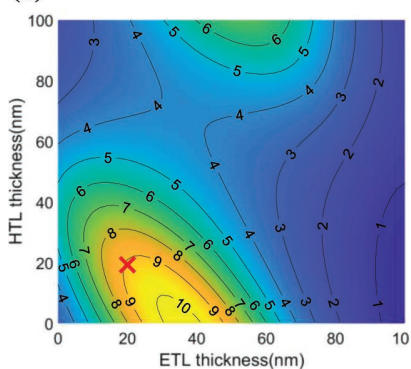

(b)

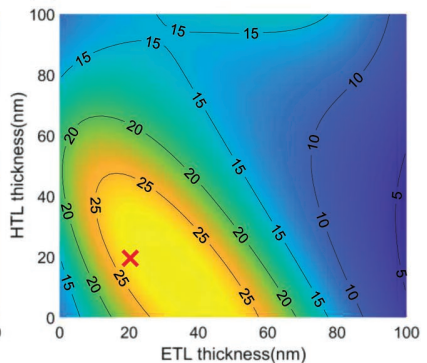

(c)

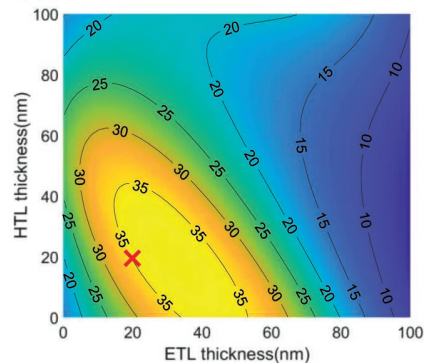

Figure 5. Simulations of the external quantum efficiencies of a) CzTrz, b) BCzTrz, and c) TCzTrz, versus hole (HTL) and electron transport layer (ETL) thicknesses of the OLEDs. The experimental OLEDs are indicated by "X."

TCzTrz devices were $(0.17,0.11),(0.23,0.42)$, and $(0.20,0.44)$, respectively. Performances of the CzTrz, BCzTrz, and TCzTrz OLEDs are summarized in Table 2.

\section{Conclusions}

We have demonstrated a direct correlation between the molecular structure of the donor moiety of vacuum deposited TADF emitting molecules with their anisotropic dipole orientation in OLEDs in which they were employed. Triscarbazole donor units were shown to be effective in inducing a nearly perfectly aligned horizontal dipole relative to the substrate plane. Moreover, the triscarbazole donor unit results in nearly complete upconversion of triplet to singlet excitons. As a result of these factors, the triscarbazole donor-based TCzTrz TADF emitter showed EQE $=31.8 \% \pm 0.31 \%$ when employed in OLEDs. These results point to the advantages of designing molecules with extended molecular donor moieties to achieve high efficiency through dipole alignment and high luminance efficiencies for emission across the visible spectrum.

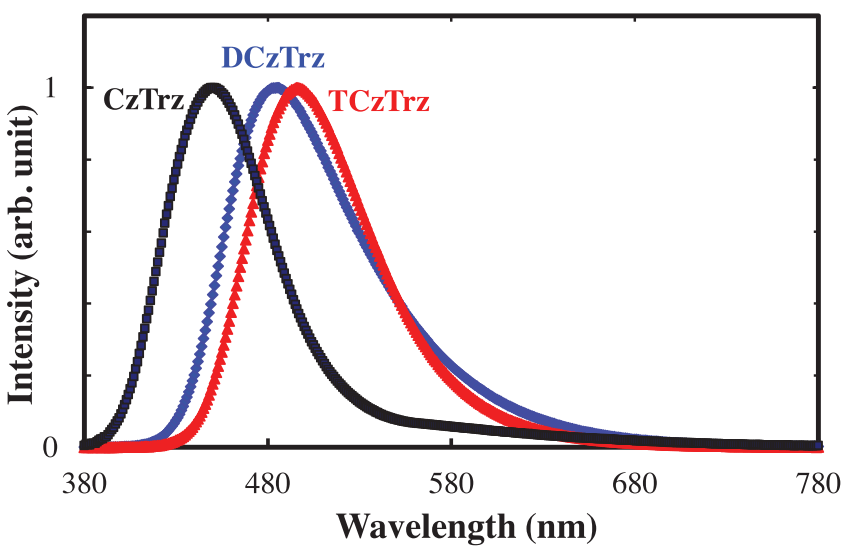

Figure 6. Electroluminescence spectra of CzTrz, BCzTrz, and TCzTrzbased OLEDs at a luminance of $1000 \mathrm{~cd} \mathrm{~m}^{-2}$. Doping concentration of the TADF emitters is $10 \mathrm{wt} \%$ in DPEPO.

\section{Experimental Section}

General Information: 2-Chloro-4,6-diphenyl-1,3,5-triazine, 3-bromo$9 \mathrm{H}$-carbazole, and 3,6-dibromo-9H-carbazole were purchased from Sun Fine Clobal Co. Ltd., tetrakis(triphenylphosphine)palladium (0) and (9-phenyl-9H-carbazol-3-yl)boronic acid from P\&H tech Co., sodium hydride and (4-fluorophenyl)boronic acid from Alfa Aesar Co., $9 \mathrm{H}$-carbazole and dimethylsulfoxide- $\mathrm{d}_{6}$ from Sigma-Aldrich Co. LLC., N,N-dimethylformamide (DMF, from Duksan Sci. Co.), and tetrahydrofuran (from Samchun pure chemical Co. Ltd). Information regarding analytic methods was provided previously. ${ }^{[30]}$

Synthesis: $\quad$ 9'-(4-(4,6-diphenyl-1,3,5-triazin-2-yl)phenyl)-9, 9"-diphenyl$9 H, 9^{\prime} H, 9^{\prime \prime} H-3,3^{\prime}: 6^{\prime}, 3^{\prime \prime}$-tercarbazole (TCzTrz) (Scheme 1): Sodium hydride $(0.09 \mathrm{~g}, 0.23 \mathrm{mmol})$ was poured into a round-bottomed flask and stirred after vacuum drying for $2 \mathrm{~h}$. 9"-Diphenyl-9H, $9^{\prime} H, 9^{\prime \prime} H-3,3^{\prime}: 6^{\prime}, 3^{\prime \prime}$ tercarbazole $(1.00 \mathrm{~g}, 0.15 \mathrm{mmol})$ was dissolved in DMF $(6 \mathrm{~mL})$, and slowly injected into the reaction vessel. 2-(4-fluorophenyl)-4,6-diphenyl1,3,5-triazine $(0.40 \mathrm{~g}, 0.23 \mathrm{mmol})$ dissolved in DMF $(10 \mathrm{~mL})$ was slowly added to the reaction and stirred for $1 \mathrm{~h}$. The reaction was quenched using distilled water, and the yellow-colored precipitate was filtered. The

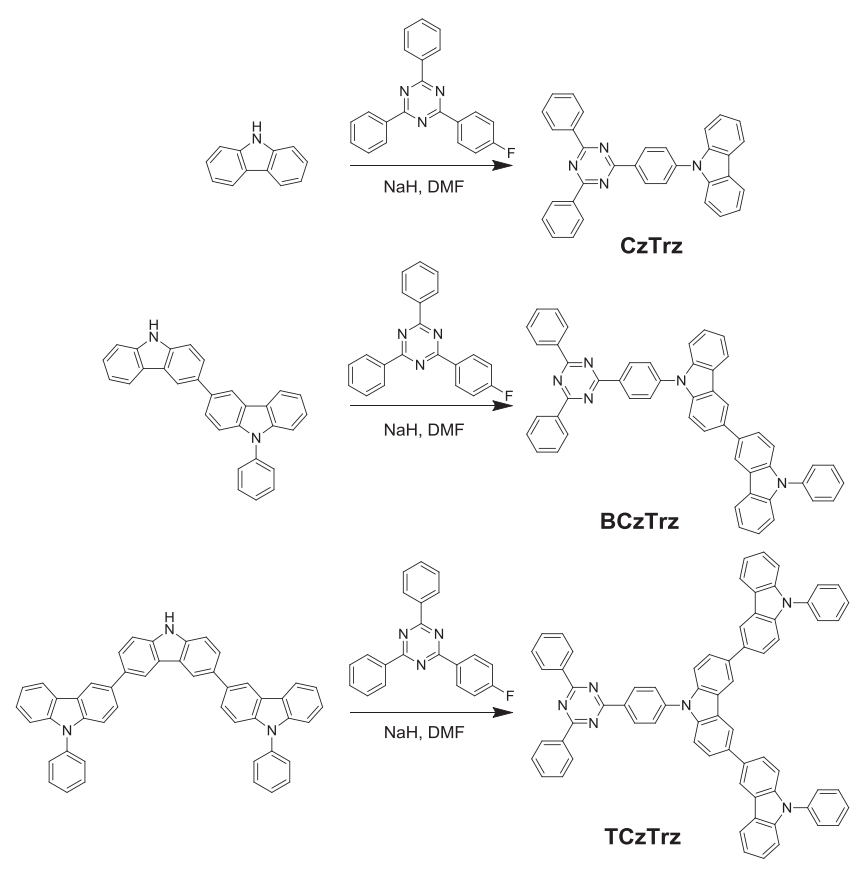

Scheme 1. Synthetic scheme of CzTrz, BCzTrz, and TCzTrz. 
Table 2. Summary of performances of CzTrz-, BCzTrz-, and TCzTrz-based OLEDs.

\begin{tabular}{|c|c|c|c|c|c|c|c|c|}
\hline & \multicolumn{2}{|c|}{ External quantum efficiency [\%] } & \multicolumn{2}{|c|}{ Power efficiency $\left[\mathrm{Im} \mathrm{W} \mathrm{W}^{-1}\right]$} & \multicolumn{2}{|c|}{ Current efficiency $\left[c d A^{-1}\right]$} & \multicolumn{2}{|c|}{ Color coordinates } \\
\hline & {$\left[1000 \mathrm{~cd} \mathrm{~m}^{-2}\right]$} & {$[\mathrm{Max}]$} & {$\left[1000 \mathrm{~cd} \mathrm{~m}^{-2}\right]$} & {$[\mathrm{Max}]$} & {$\left[1000 \mathrm{~cd} \mathrm{~m}^{-2}\right]$} & {$[\mathrm{Max}]$} & $x$ & $y$ \\
\hline CzTrz & 0.6 & 4.2 & 0.1 & 2.7 & 0.53 & 3.8 & 0.17 & 0.11 \\
\hline BCzTrz & 5.2 & 23.6 & 3.5 & 46.6 & 11.8 & 53.3 & 0.23 & 0.42 \\
\hline TCzTrz & 11.3 & 31.8 & 10.0 & 61.5 & 39.4 & 86.4 & 0.20 & 0.44 \\
\hline
\end{tabular}

precipitate was further purified by column chromatography using an eluent of MC and HEX 1:1 (0.8 g, yield 55\%).

${ }^{1} \mathrm{H}$ NMR $\left(500 \mathrm{MHz}\right.$, DMSO-d 6 ) $\delta 8.22\left(\mathrm{~d}, 2 \mathrm{H}, \mathrm{J}=7.0 \mathrm{H}_{\mathrm{z}}\right), 7.71(\mathrm{t}$, $\left.4 \mathrm{H}, \mathrm{J}=8.25 \mathrm{H}_{\mathrm{z}}\right), 7.43-7.32(\mathrm{~m}, 14 \mathrm{H}), 7.27\left(\mathrm{t}, 4 \mathrm{H}, \mathrm{J}=7.5 \mathrm{H}_{\mathrm{z}}\right) .{ }^{13} \mathrm{C} \mathrm{NMR}$ $\left(125 \mathrm{MHz}, \mathrm{CDCl}_{3}\right) 158.3,140.8,139.5,131.3,126.3,123.7,122.4,120.5$, 120.4, 118.0, 117.8, 109.9. MS (APCI) $\mathrm{m} / \mathrm{z} 958.0\left[(\mathrm{M}+\mathrm{H})^{+}\right]$.

Device Fabrication and Measurements: The OLED structure is indium tin oxide $(50 \mathrm{~nm}) /$ PEDOT:PSS $(60 \mathrm{~nm}) /$ TAPC $(20 \mathrm{~nm}) / \mathrm{mCP}(10 \mathrm{~nm}) /$ emitting layer $(25 \mathrm{~nm}) /$ TSPOI $(5 \mathrm{~nm}) /$ TBPi $(20 \mathrm{~nm}) / \mathrm{LiF}(1.5 \mathrm{~nm}) / \mathrm{Al}$ $(200 \mathrm{~nm})$, where PEDOT:PSS is poly (3,4-ethylenedioxythiophene):poly(st yrenesulfonate), TAPC was 1,1-bis [(di-4-tolylamino) phenyl] cyclohexane, $\mathrm{mCP}$ is 1,3-di(9H-carbazol-9-yl)benzene, TSPO1 is diphenylphosphine oxide-4-(triphenylsilyl), and TPBi is 1,3,5-tris-(2-N-phenylbenzimidazolyl) benzene. The emitting layer comprises a DPEPO film doped with 10 wt\% TADF molecules. All devices were fabricated under high vacuum $\left(<1.0 \times 10^{-7}\right.$ Torr). Current density-voltage-luminance $(U-V-L)$ characteristics were carried out using an electrical and photometric measurement system equipped with a Keithley 2400 sourcemeter (Keithley Instruments, Inc) for J-V measurement and a CS 2000 (Konica Minolta Inc.) spectroradiometer.

Optical Measurements: Alignments of the transition dipole moments are inferred from angle dependent p-polarized emission. ${ }^{[31,32]}$ The substrate was placed perpendicular to the plane of detection and the emission was outcoupled from the substrate using a $2 \mathrm{~cm}$ radius, half-cylindrical lens. The emission along the plane of detection was decomposed into transverse electric and magnetic modes using a polarization analyzer. A motorized stage was used to position the detector. Simulations of the angular intensity profiles are based on the dyadic Green's function in a birefringent medium. ${ }^{[28]}$ A least-squares algorithm was used to fit the experimental data to the simulation. The refractive indices and extinction coefficients of materials were measured using variable-angle spectroscopic ellipsometry.

\section{Supporting Information}

Supporting Information is available from the Wiley Online Library or from the author.

\section{Acknowledgements}

S.Y.B. and J.K. contributed equally to this work.

\section{Conflict of Interest}

One of the authors (S.R.F.) has an equity interest in Universal Display Corp.

\section{Keywords}

blue devices, delayed fluorescence, donor units, triscarbazole, upconversion efficiency

Received: December 9, 2017 Revised: March 22, 2018 Published online: May 22, 2018
[1] H. Uoyama, K. Goushi, K. Shizu, H. Nomura, C. Adachi, Nature 2012, 492, 234.

[2] J. Li, T. Nakagawa, J. MacDonald, Q. Zhang, H. Nomura, H. Miyazaki, C. Adachi, Adv. Mater. 2013, 25, 3319.

[3] L. Q. Meng, H. Wang, X. F. Wei, J. J. Liu, Y. Z. Chen, X. B. Kong, X. P. Lv, P. F. Chen, Y. Wang, ACS Appl. Mater. Interfaces 2016, 8, 20955.

[4] M. Taneda, K. Shizu, H. Tanaka, C. Adachi, Chem. Commun. 2015, $51,5028$.

[5] Q. S. Zhang, B. Li, S. P. Huang, H. Nomura, H. Tanaka, C. Adachi, Nat. Photonics 2014, 8, 326.

[6] D. R. Lee, B. S. Kim, C. W. Lee, Y. Im, K. S. Yook, S. H. Hwang, J. Y. Lee, ACS Appl. Mater. Interfaces 2015, 7, 9625.

[7] T.-A. Lin, T. Chatterjee, W.-L. Tsai, W.-K. Lee, M.-J. Wu, M. Jiao, K.-C. Pan, C.-L. Yi, C.-L. Chung, K.-T. Wong, C.-C. Wu, Adv. Mater. 2016, 28, 6976

[8] Q. Zhang, J. Li, K. Shizu, S. Huang, S. Hirata, H. Miyazaki, C. Adachi, J. Am. Chem. Soc. 2012, 134, 14706.

[9] H. Yang, Q. Q. Liang, C. M. Han, J. Zhang, H. Xu, Adv. Mater. 2017, 29, 1700553.

[10] J. Zhang, D. X. Ding, Y. Wei, F. Q. Han, H. Xu, W. Huang, Adv. Mater. 2016, 28, 479.

[11] D. R. Lee, M. Kim, S. K. Jeon, S-H. Hwnag, C. W. Lee, J. Y. Lee, Adv. Mater. 2015, 27, 5861.

[12] M. Kim, S. K. Jeon, S.-H. Hwang, J. Y. Lee, Adv. Mater. 2015, 27, 2515.

[13] S. Hirata, Y. Sakai, K. Masui, H. Tanaka, S. Y. Lee, H. Nomura, N. Nakamura, M. Yasumatsu, H. Nakanotani, Q. S. Zhangm, K. Shizu, H. Miyazaki, C. Adachi, Nat. Mater. 2015, 14, 330.

[14] I. Lee, J. Y. Lee, Org. Electron. 2016, 29, 160.

[15] K. C. Pan, S. W. Li, Y. Y. Ho, Y. J. Shiu, W. L. Tsai, M. Jiao, W. K. Lee, C. C. Wu, C. L. Chung, T. Chatterjee, Y. S. Li, K. T. Wong, H. C. Hu, C. C. Chen, M. T. Lee, Adv. Funct. Mater. 2016, 26, 7560.

[16] a) C. Mayr, S. Y. Lee, T. D. Schmidt, T. Yasuda, C. Adachi, W. Brutting, Adv. Funct. Mater. 2014, 24, 5232; b) K. Tuong Ly, Y. Chi, Nat. Photonics 2017, 11, 63; c) K-H. Kim, J-J. Kim, Adv. Mater. 2016, 28, 2526.

[17] C. S. Oh, C.-K. Moon, J. M. Choi, J.-S. Huh, J.-J. Kim, J. Y. Lee, Org. Electron. 2017, 42, 337.

[18] D. H. Kim, K. Inada, L. Zhao, T. Komino, N. Matsumoto, J. C. Ribierre, C. Adachi, J. Mater. Chem. C 2017, 5, 1216.

[19] C. M. Han, Y. B. Zhao, H. Xu, J. S. Chen, Z. P. Deng, D. G. Ma, Q. Li, P. F. Yan, Chem. Eur. J. 2011, 17, 5800.

[20] K. Shizu, H. Noda, H. Tanaka, M. Taneda, M. Uejima, T. Sato, K. Tanaka, H. Kaji, C. Adachi, J. Phys. Chem. C 2015, 119, 26283.

[21] M. Flammich, J. Frischeisen, D. S. Setz, D. Michaelis, B. C. Krummacher, T. D. Schmidt, W. Brutting, N. Danz, Org. Electron. 2011, 12, 1663.

[22] S.-Y. Kim, W.-I. Jeong, C. Mayr, Y.-S. Park, K.-H. Kim, J.-H. Lee, C.-K. Moon, W. Brutting, J.-J Kim, Adv. Funct. Mater. 2013, 23, 3896.

[23] J. W. Sun, J.-H. Lee, C.-K. Moon, K.-H. Kim, H. Shin, J.-J. Kim, Adv. Mater. 2014, 26, 5684.

[24] K.-H. Kim, C.-K. Moon, J.-H. Lee, S.-Y. Kim, J.-J. Kim, Adv. Mater. 2014, 26, 3844. 
[25] A. Graf, P. Liehm, C. Murawski, S. Hofmann, K. Leo, M. C. Gather, J. Mater. Chem. C 2014, 2, 10298.

[26] J. Y. Kim, D. Yokoyama, C. Adachi, J. Phys. Chem. C 2012, 116, 8699.

[27] T. Komino, Y. Sagara, H. Tanaka, Y. Oki, N. Nakamura, H. Fujimoto C. Adachi, Appl. Phys. Lett. 2016, 108, 241106.

[28] K. Celebi, T. D. Heidel, M. A. Baldo, Opt. Express 2007, 15, 1762.
[29] R. R. Chance, A. Prock, R. Silbey, J. Chem. Phys. 1974, 60, 2744.

[30] S. Y. Byeon, J. H. Kim, J. Y. Lee, ACS Appl. Mater. Interfaces 2017, 9, 13339.

[31] J. Frischeisen, D. Yokoyama, C. Adachi, W. Brütting, Appl. Phys. Lett. 2010, 96, 73302

[32] C.-K. Moon, S.-Y. Kim, J.-H. Lee, J.-J. Kim, Opt. Express 2015, 23, A279. 